\title{
Briófitas (Bryophyta e Marchantiophyta) das cangas da Serra dos Carajás, Pará, Brasil
}

Bryophytes (Bryophyta and Marchantiophyta) of the canga of the Serra dos Carajás, Pará, Brazil

\author{
Fúvio Rubens Oliveira-da-Silva ${ }^{1} \&$ Anna Luiza Ilkiu-Borges ${ }^{2,3}$
}

\begin{abstract}
Resumo
Os estudos botânicos na Serra dos Carajás iniciaram no final da década de 60. Após isso, foram publicados vários trabalhos sobre florística, ecologia e fitogeografia que resultaram em muitas descobertas sobre a flora local, culminando com a produção da flora das cangas da Serra dos Carajás. Este trabalho objetiva apresentar a riqueza das briófitas estudadas durante o projeto Flora das cangas da Serra dos Carajás, Pará, Brasil. O material estudado incluiu novas coletas realizadas de 2015 a 2017 e material depositado no Herbário MG. A canga apresentou uma grande riqueza de briófitas (54 musgos e 35 hepáticas) e a composição está de acordo com o esperado para áreas rupestres na Amazônia. A elevada riqueza de briófitas de sombra demonstrou que as poucas árvores da canga assim com as pequenas áreas de mata baixa que formam ilhas de vegetação ou ainda nichos específicos como frestas e base de rochas ou espaços sob rochas, servem como refúgio para essas espécies. O estudo das briófitas na Serra dos Carajás possibilitou o conhecimento 51 novos registros para as cangas de Carajás, sete para o Pará, três para a região Norte e um para o Brasil. Palavras-chave: Musgos, hepáticas, afloramentos rochosos, campos rupestres.
\end{abstract}

\begin{abstract}
The Botanical studies in Serra dos Carajás initiated in the late 60's. Thereafter, several papers on floristics, ecology and phytogeography were published, which resulted in many discoveries about the local flora, culminating in the production of the flora of the canga of the Serra dos Carajás. This work aims to present the richness of the bryophytes studied during the Flora of the canga of the Serra dos Carajás, Pará, Brazil. The studied material included new collections from 2015 to 2017 and specimens deposited in Herbarium MG. The canga presented a high bryophyte richness (54 mosses and 35 liverworts) and the composition was the expected for rupestrian areas in Amazonia. The high richness of shade specialists has shown that the few trees in the canga as well as the small areas of low forests forming vegetation islands or even specific niches such as cracks and base of rocks or spaces under rocks, serve as refuge for these species. The study of the bryophytes in the Serra dos Carajás made it possible to discover 51 new records for the canga of Carajás, seven for Pará, three for the North Brazilian region, and one for Brazil.
\end{abstract}

Key words: Mosses, liverworts, rocky outcrops, rupestrian grasslands.

\section{Introdução}

Os estudos botânicos na Serra dos Carajás iniciaram no final da década de 60, quando pesquisadores do Museu Paraense Emílio Goeldi (MPEG) realizaram expedições na região. A partir disso, foram publicados vários estudos florísticos, ecológicos e fitogeográficos que resultaram em grandes descobertas sobre a flora da Serra dos Carajás (Viana et al. 2016).
O conhecimento sobre a brioflora na Serra dos Carajás iniciou com a publicação de Lisboa (1994), que tratava sobre novas ocorrências para o estado do Pará, incluindo quatro espécies coletas no município de Parauapebas (Serra dos Carajás) entre os anos de 1992 a 1993, a saber: Rhodobryum subverticillatum Broth., Porotrichum plicatulum Mitt., Lepidopilum scabrisetum (Schwaegr.) Steere, Lepidopilum stolonaceum C. Müll. Duas dessas espécies foram sinonimizadas por Churchill (1998).

\footnotetext{
${ }^{1}$ Universidade Federal Rural da Amazônia/Museu Paraense Emílio Goeldi, Pgm. Pós-graduação em Ciências Biológicas - Botânica Tropical, Av. Perimetral 1901, 66530-070, Belém, PA, Brasil.

${ }^{2}$ Museu Paraense Emílio Goeldi, Av. Magalhães Barata 376, São Braz, 66040-170, Belém, PA, Brasil.

${ }^{3}$ Autor para correspondência: ilkiu-borges@museu-goeldi.br
} 
Posteriormente, Lisboa \& Ilkiu-Borges (1996) identificaram as briófitas de diferentes ecossistemas com objetivo de associá-las a determinados depósitos minerais como ouro, ferro, níquel, manganês e cobre. No total, foram registradas 20 famílias, sendo 17 de musgos (57 espécies e duas variedades) e três de hepáticas (três espécies). Das espécies identificadas, 46 foram coletadas em ambiente de canga.

Moraes \& Lisboa (2006), dando continuidade às identificações de Lisboa \& Ilkiu-Borges (1996), incluíram somente musgos em seus estudos e acrescentaram mais 30 espécies e uma variedade aos já relacionados anteriormente, totalizando 87 espécies e três variedades registradas em vários ecossistemas da Serra dos Carajás. Desses registros, 49 haviam sido coletados em canga. As hepáticas não foram estudadas, ficando o conhecimento desse grupo restrito às três espécies citadas em Lisboa \& Ilkiu-Borges (1996): Mastigolejeunea auriculata (Wilson) Schiffn., Calypogeia lechleri Mont. e Lophocolea martiana Nees.

Os trabalhos mais recentes realizados nas Serras dos Carajás foram os de Oliveira-da-Silva \& Ilkiu-Borges (2016a,b; 2017a,b,c,d) e Ilkiu-Borges \& Oliveira-da-Silva (2016a,b; 2017a,b,c,d), frutos do projeto "Flora das cangas da Serra dos Carajás, Pará, Brasil", uma parceria estabelecida em 2014, entre o Museu Paraense Emílio Goeldi (MPEG) e o Instituto Tecnológico Vale de Desenvolvimento Sustentável (ITVDS). O projeto contou com a participação de diversos pesquisadores do Brasil e do mundo, e todos os tratamentos da flora apresentaram ilustrações, comentários morfológicos, chaves de identificação e descrição das famílias, gêneros e espécies de angiospermas, gimnospermas, licófitas, samambaias e briófitas coletadas em canga.

Os artigos produzidos aprofundaram ainda mais o conhecimento sobre a flora da Serra dos Carajás, mais precisamente das áreas de canga, que são formações rupestres ferríferas que se distribuem como platôs de altitude entre 600 a $800 \mathrm{~m}$, e possuem uma vegetação altamente especializada, com espécies endêmicas e elevada riqueza vegetal (Cavalcante 1970; Secco \& Mesquita 1983; Silva 1991; Silva et al. 1996; Lisboa \& Ilkiu-Borges 1996; Moraes \& Lisboa 2006; Mota et al. 2015; Viana et al. 2016).

O objetivo deste trabalho é apresentar a riqueza (lista completa) das briófitas estudadas durante o projeto Flora das cangas da Serra dos Carajás, Pará, Brasil.

\section{Material e Métodos}

A área de estudo compreende a Serra dos Carajás ( $5^{\circ} 54^{\prime}-6^{\circ} 33^{\prime} \mathrm{S}$ e $\left.49^{\circ} 53^{\prime}-50^{\circ} 34^{\prime} \mathrm{W}\right)$, que está sob o domínio da floresta amazônica, localizada no sudeste do estado do Pará, nos municípios de Parauapebas e Canaã dos Carajás (Golder 2010; Silva et al. 1996; Silva 1991; Secco \& Mesquita 1983) (Fig. 1).

A Serra dos Carajás é formada principalmente por dois grandes blocos: a Serra Norte e a Serra Sul, além de outras serras de menores dimensões. $\mathrm{Na}$ Serra Norte, os corpos de minério são organizados por blocos intitulados N1, N2, N3, N4, N5, N6, N7, N8 e N9 e na Serra Sul, identificados como S11A, S11B, S11C, S11D, Serra do Tarzan e Serra da Bocaina. A Serra do Tarzan e a Serra da Bocaina são consideradas como pertencentes a Serra Sul, apesar de disjuntas (Mota et al. 2015; Viana et al. 2016).

As maiorias das serras situam-se dentro da FLONA Carajás e formam aproximadamente 400 mil hectares. O clima, segundo a classificação de Köppen, é do tipo AWi, Tropical Chuvoso com seca de inverno, apresentando dois subtipos: o Equatorial Continental, associado a encostas e terras baixas, e o clima Equatorial Mesotérmico de Altitude, associado ao topo de serra. Este último é caracterizado pela temperatura média entre $23-25^{\circ} \mathrm{C}$ e precipitação anual variando entre 2.000 e $2.400 \mathrm{~mm}$ (STCP 2003).

Foram realizadas coletas em quatro excursões, 27 a 31 de abril de 2015, 31 de agosto a 3 de setembro de 2015, 23 a 25 de fevereiro de 2016 e 09 a 14 de maio de 2017. As amostras foram retiradas em diferentes tipos de substratos, como tronco vivo, tronco em decomposição, rocha de ferro, folha, solo e sobre cupinzeiro. A metodologia de coleta, herborização e preservação do material botânico seguiu Yano (1984).

Além das amostras coletadas nas excussões realizadas pelo projeto "Flora das cangas da Serra dos Carajás, Pará, Brasil", foram observadas e listadas exsicatas de briófitas das áreas de canga que estão depositadas no Herbário João Murça Pires (MG) do Museu Paraense Emílio Goeldi (MPEG).

As identificações foram realizadas no laboratório de Briologia do MPEG através de literaturas especializadas, como Crum \& Anderson (1981), Frahm (1991), Yano (1992), Lisboa (1993), Reese (1993), Sharp et al. (1994), Grolle \& Reiner-Drehwald (1997), Gradstein et al. (2001), Buck (2003; 2006), Gradstein \& Costa (2003), Pursel (2007), Gradstein \& Ilkiu-Borges 


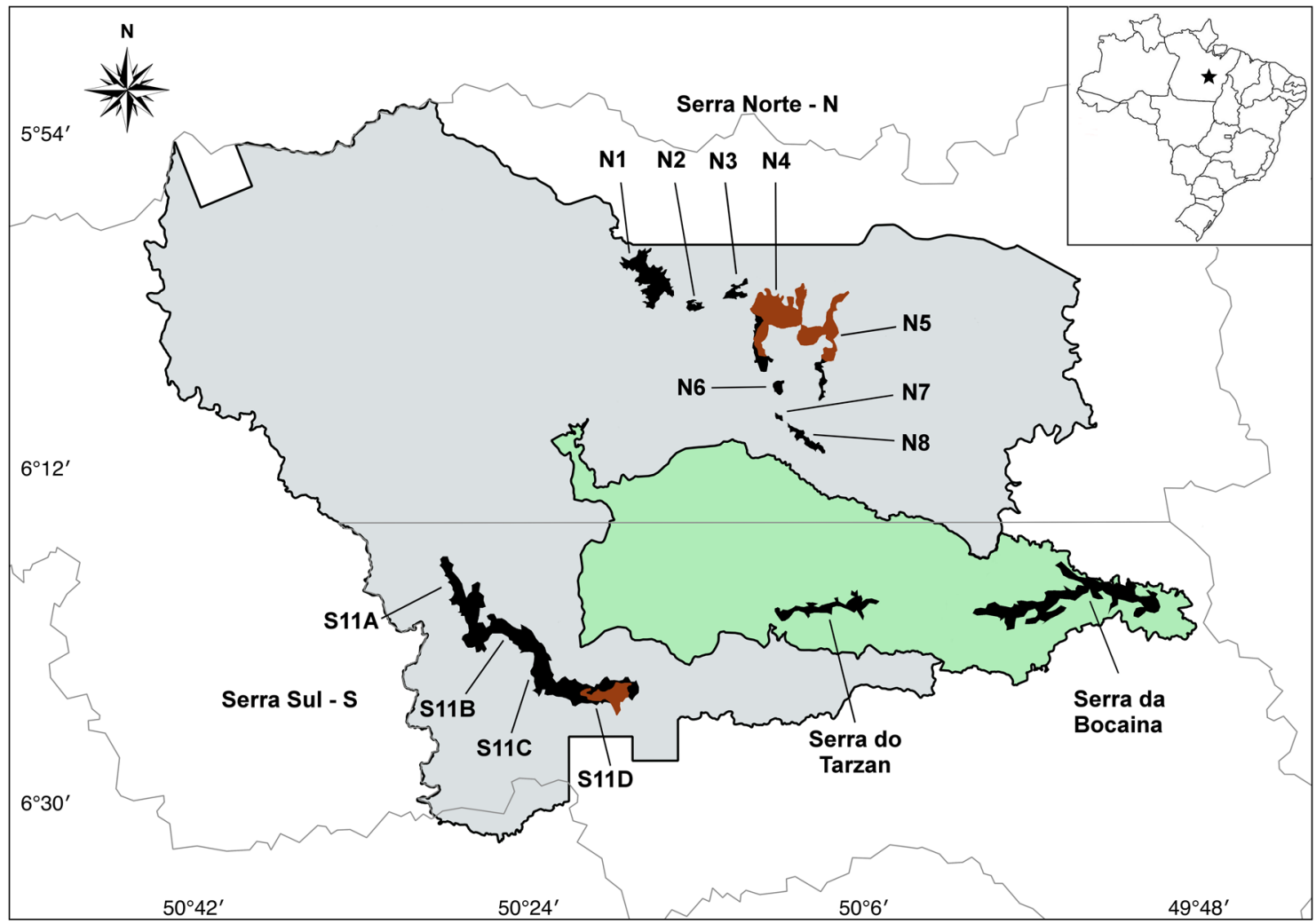

Figura 1 - Área de estudos, com indicação dos platôs de canga amostrados. Em preto, áreas de canga remanescentes; em vermelho, áreas de canga suprimidas (representação com base em imgem de satélite de dezembro de 2016, disponível no Google Earth). Em cinza claro, delimitação da Floresta Nacional de Carajás. Em verde, o Parque Nacional dos Campos Ferruginosos. Fonte: Mota et al. 2018.

Figure 1 - Study area, indicating the canga plateaux sampled. In black, remaining canga areas; in red, suppressed canga areas (representation based on satelite image taken in December 2016, available on Google Earth). In grey, Floresta Nacional de Carajás. In green, Parque Nacional dos Campos Ferruginosos. Source: Mota et al. 2018.

(2009), e entre outras. A classificação adotada para Bryophyta e Marchantiophyta está de acordo com Goffinet et al. (2008) e Crandall-Stotler et al. (2008), respectivamente. Todas as amostras serão depositadas no Herbário MG.

As espécies foram classificadas de acordo com sua tolerância à luz solar como especialistas de sol, especialistas de sombra e generalistas. Para tal, foram utilizados os trabalhos de Richards (1984), Gradstein et al. (2001), Gradstein \& Costa (2013), Gradstein \& Ilkiu-Borges (2009), Tavares-Martins (2009), Santos et al. (2011), Visnadi (2013) e Fagundes et al. (2016).

A distribuição geográfica brasileira e mundial das espécies foi baseada nas informações disponíveis no trabalho Synopsis of the Brazilian moss flora: checklist, synonyms, distribution and conservation (Costa et al. 2011), BFG (2015), Gradstein \& Costa (2013) e Yano (2011).

\section{Resultados e Discussão}

Foram analisadas 736 exsicatas de briófitas das áreas de canga da Serra dos Carajás, sendo 452 resultantes de excussões deste projeto e 284 que estão depositadas no Herbário MG. No total, foram identificados 1282 espécimes de briófitas, sendo 852 musgos (568 deste projeto e 284 depositados no herbário MG) e 430 hepáticas. Os musgos estão distribuídos em 54 espécies, 28 gêneros e 14 famílias, enquanto as hepáticas apresentaram 35 espécies, 18 gêneros e oito famílias (Tab. 1).

As cangas da Serra dos Carajás apresentaram uma grande diversidade e riqueza de briófitas (Fig. 2) quando comparada com estudos realizados anteriormente na Serra dos Carajás (Lisboa \& Ilkiu-Borges 1996; Moraes \& Lisboa 2006) e em afloramentos rochosos no Brasil, como em sete áreas no estado de Pernambuco 


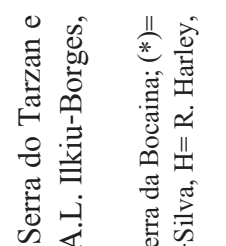

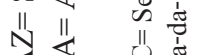

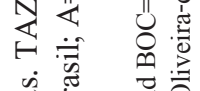

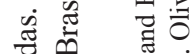

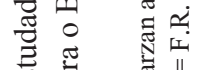

苟 䨔 类

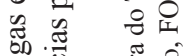

चु एँ

急

क

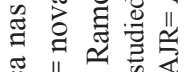

苋

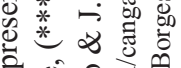

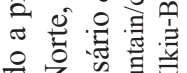

娄获言

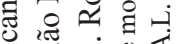

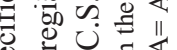

क्षे $\approx 110$

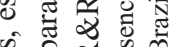

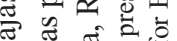

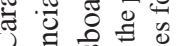

क :

تृ

क जै की

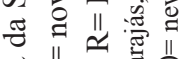

范

卷莫的 *

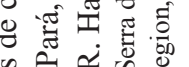

幽

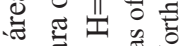

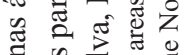

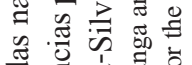

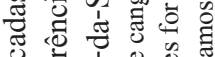

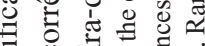

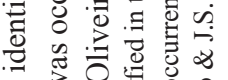

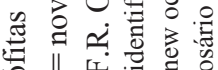

: 11 项:

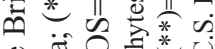

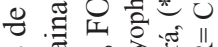

क ठ ह

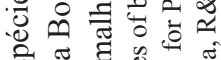

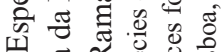

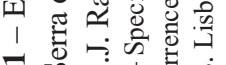

च \&

步

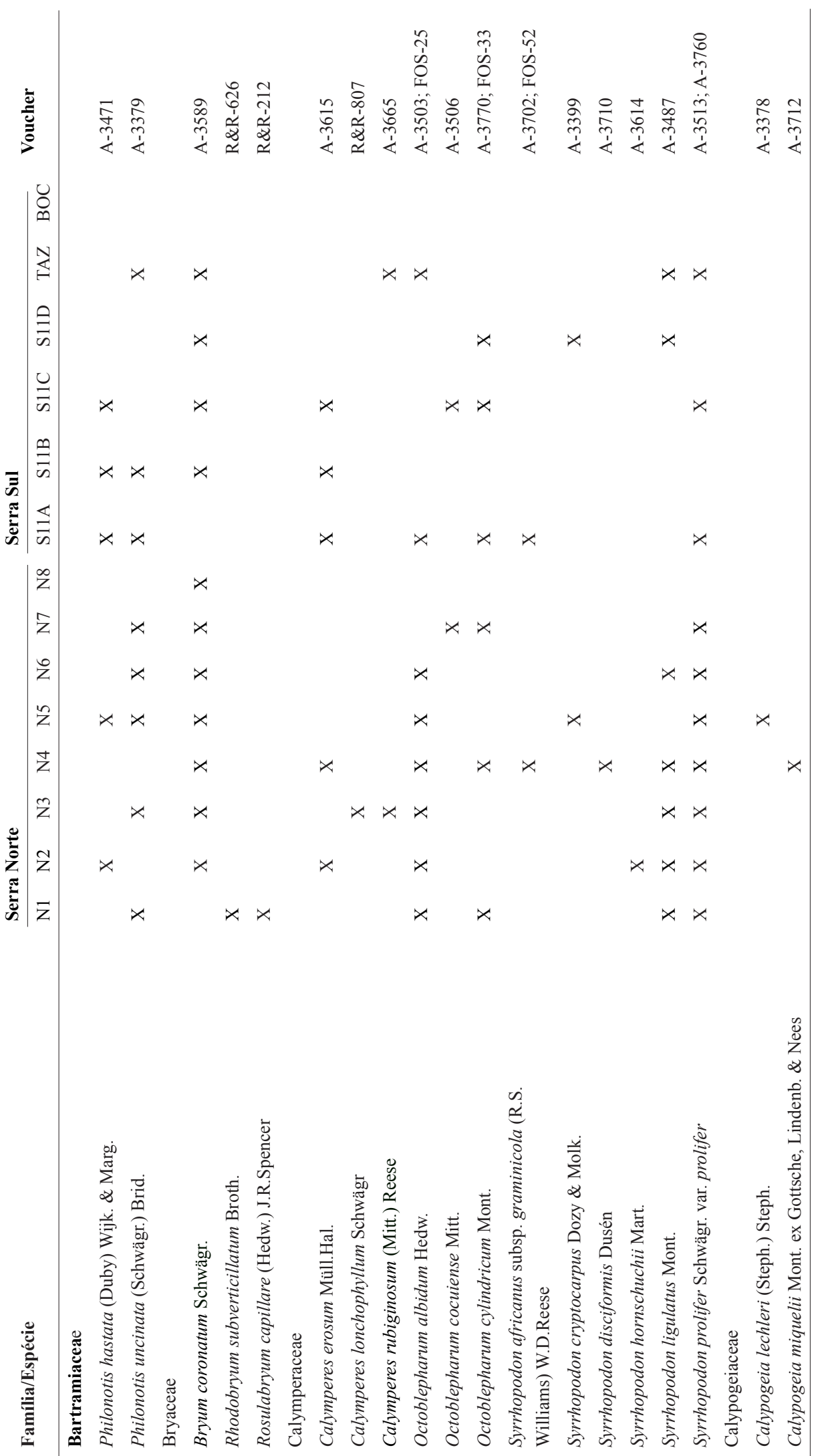




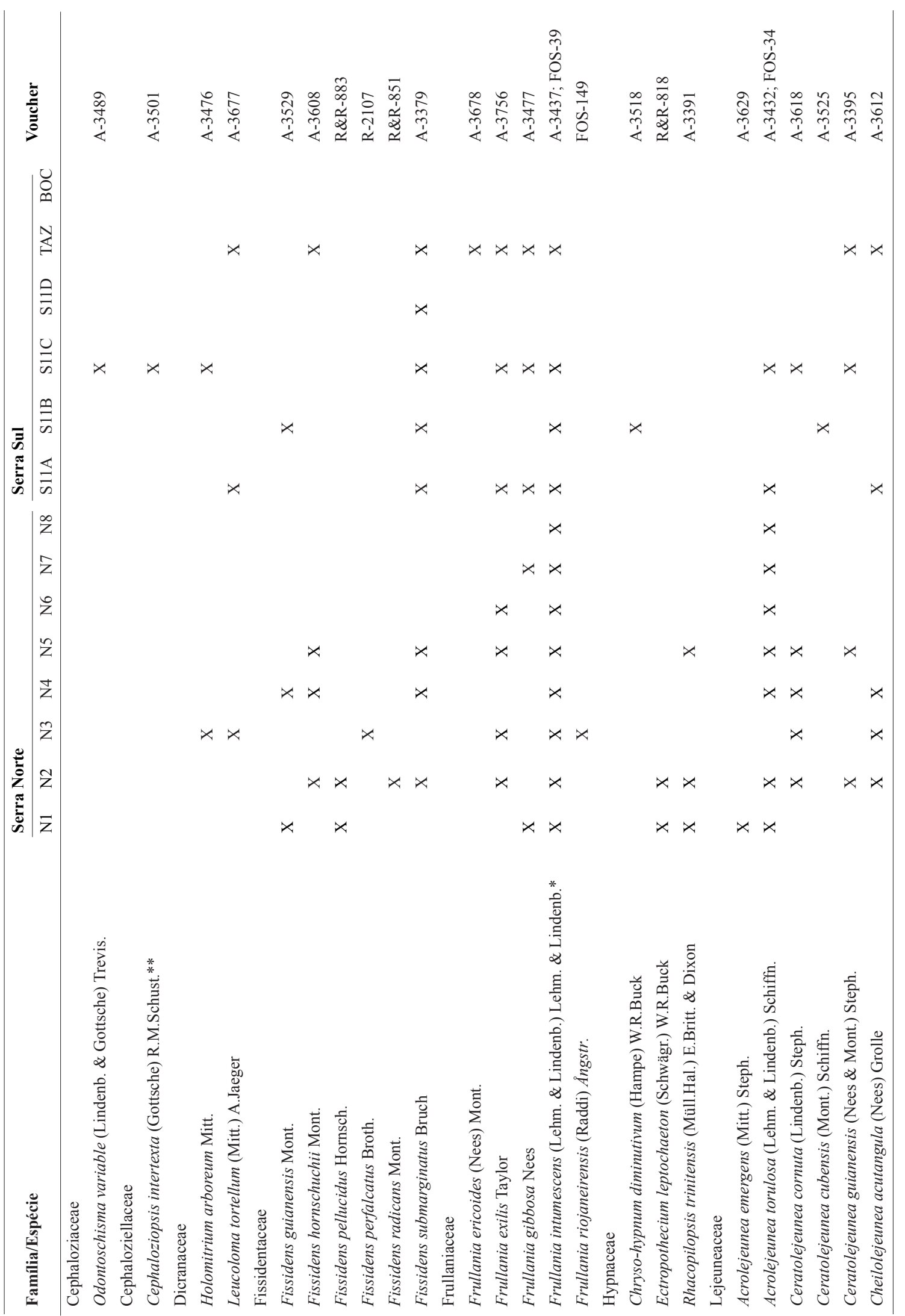




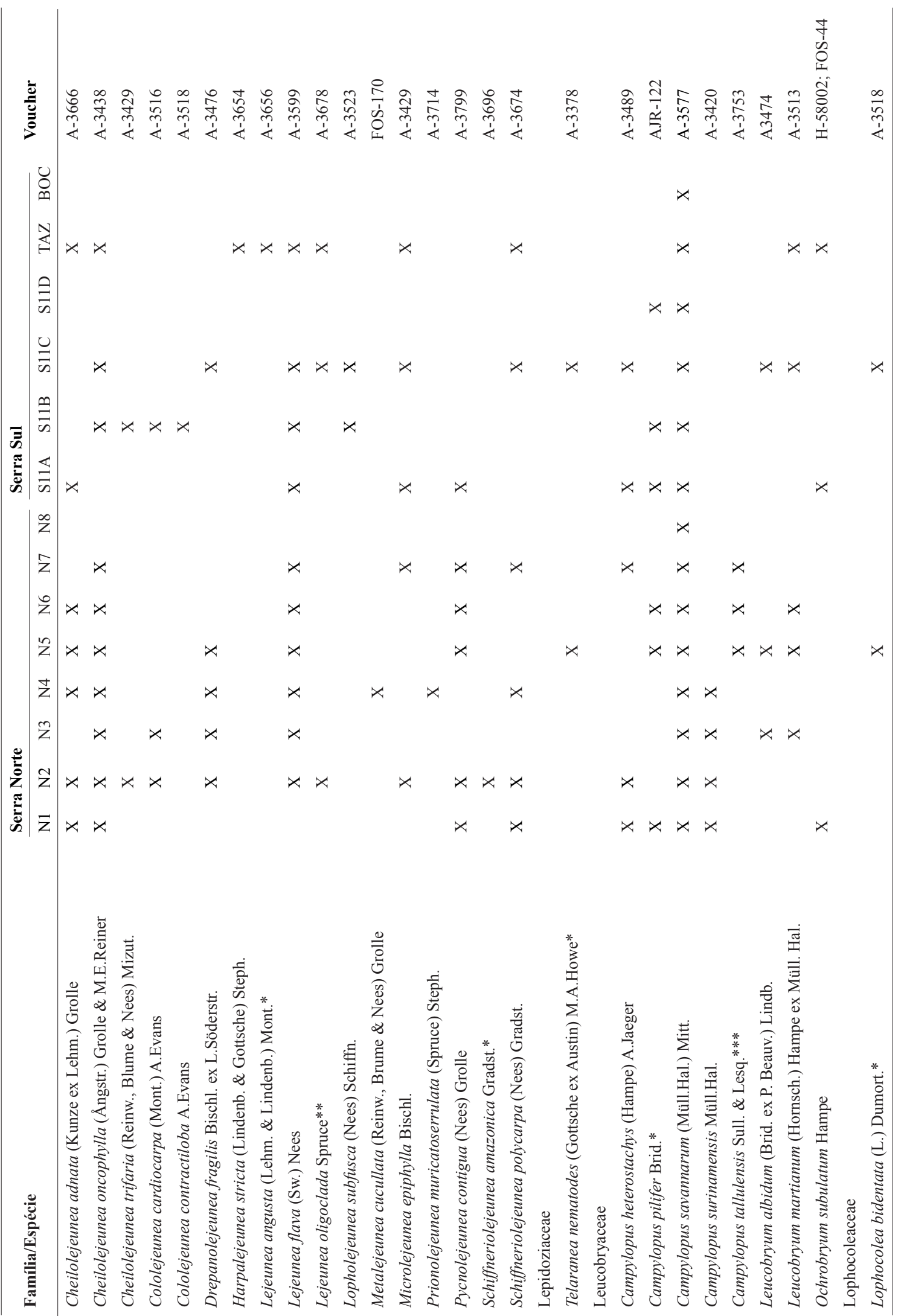


Briófitas de Carajás

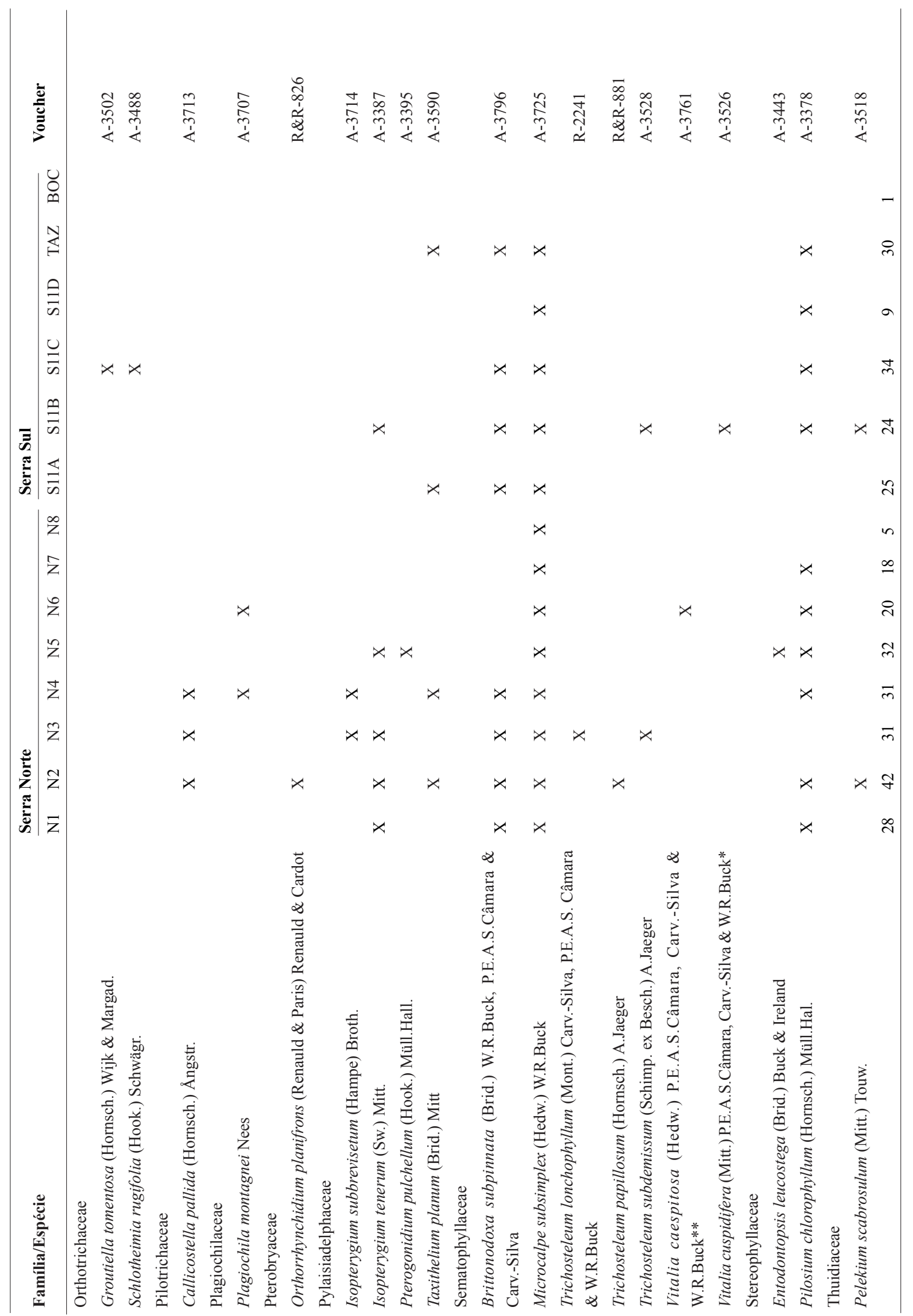



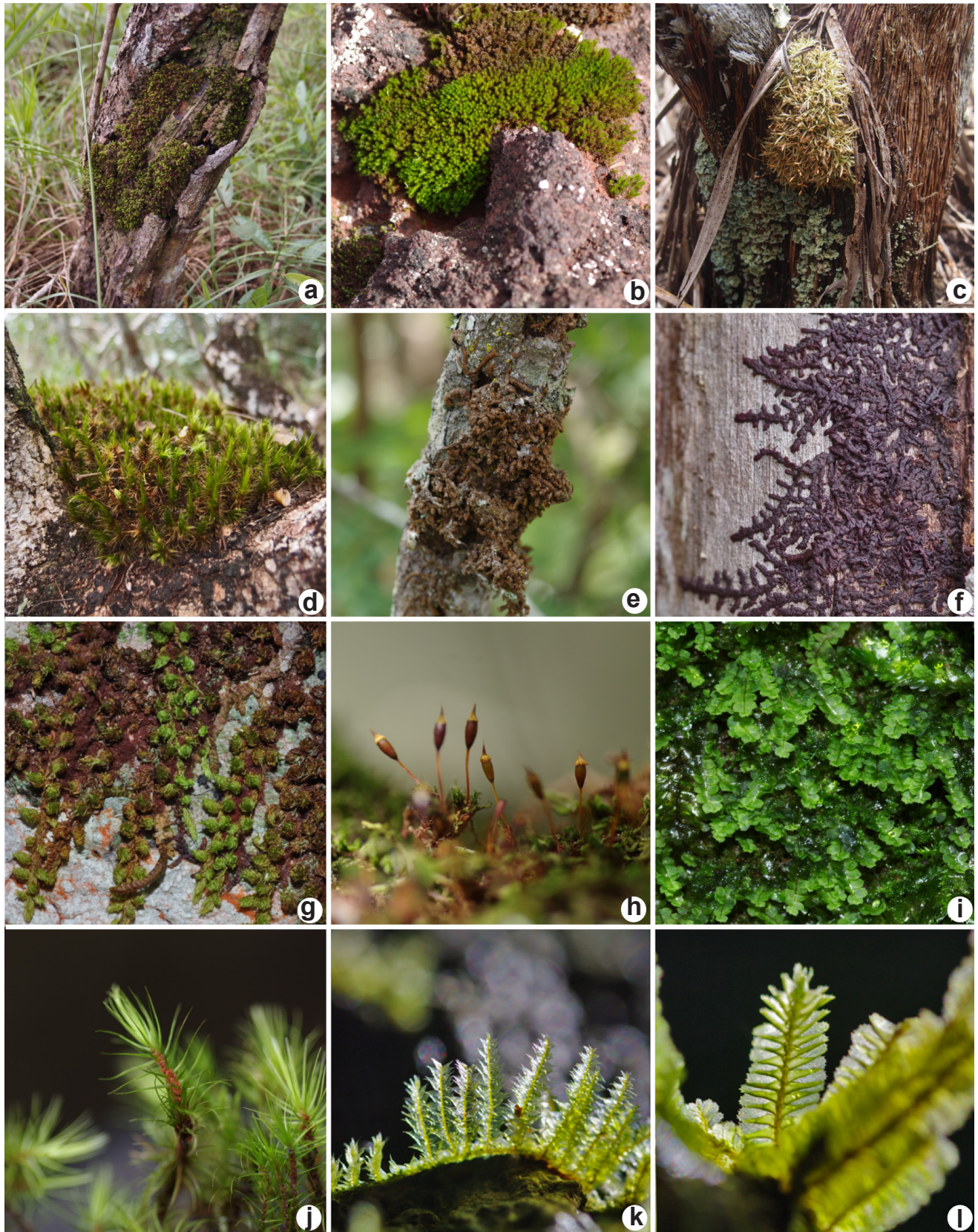

Figura 2 - Diversidade de substratos e espécies de briófitas das cangas da Serra dos Carajás - a. Bryum coronatum Schwägr. sobre madeira em decoposição; b. Philonotis uncinata (Schwägr.) Brid. sobre rocha de ferro; c. Octoblepharum cylindricum Mont. sobre tronco vivo; d. Campylopus savannarum (Müll. Hal.) Mitt. sobre tronco vivo; e. Acrolejeunea torulosa (Lehm. et Lindenb.) Schiffn. sobre tronco vivo; f. Frullania intumescens (Lehm. \& Lindenb.) Lehm. \& Lindenb. sobre tronco vivo; g-h. Schlotheimia rugifolia (Hook.) Schwägr. sobre tronco vivo; i. Calypogeia lechleri (Stephani) Stephani. sobre rocha de ferro; j. Campylopus savannarum (Müll. Hal.) Mitt.; k. Isopterygium tenerum (Sw.) Mitt.; 1. Plagiochila montagnei Nees. Fotos: a, d. F.R. Oliveira-da-Silva; b, c. A.L. Ilkiu-Borges; g-h, 1. N.F.O. Mota; e-f, i, k-1. P.L. Viana. Figure 2 - Diversity of substrates and species of bryophytes from the canga of the Serra dos Carajás - a. Bryum coronatum Schwägr. on decaying wood; b. Philonotis uncinata (Schwägr.) Brid. on iron rock; c. Octoblepharum cylindricum Mont. on live trunk; d. Campylopus savannarum (Müll. Hal.) Mitt. on live trunk; e. Acrolejeunea torulosa (Lehm. et Lindenb.) Schiffn. on live trunk; f. Frullania intumescens (Lehm. \& Lindenb.) Lehm. \& Lindenb. on live trunk; g-h. Schlotheimia rugifolia (Hook.) Schwägr. on live trunk; i. Calypogeia lechleri (Stephani) Stephani. on iron rock; j. Campylopus savannarum (Müll. Hal.) Mitt.; k. Isopterygium tenerum (Sw.) Mitt.; 1. Plagiochila montagnei Nees. Photos: a, d. F.R. Oliveira-da-Silva; b, c. A.L. Ilkiu-Borges; g-h, 1. N.F.O. Mota; e-f, i, k-1. P.L. Viana. 
(Silva et al. 2014), na Chapada Diamantina, Bahia e Minas Gerais (Valente et al. 2017) e no Parque do Rola Moça e Parque Nacional Gandarela, Minas Gerais (Peñaloza-Bocajá et al. 2017) (Fig. 3).

As famílias mais representativas foram Lejeuneaceae (23 spp./ 308 ocorrências), Calymperaceae (12 spp./ 129 ocorrências), Sematophyllaceae (8 spp./ 122 ocorrências) e Leucobryaceae (7 spp./ 136 ocorrências). Juntas essas famílias representam mais de $70 \%$ da riqueza de briófitas encontrada nas cangas (Fig. 4). Mesmo sendo um grupo típico de florestas, Lejeuneaceae se destacou na canga, justificando sua importância e abundancia na região neotropical (Gradstein et al. 2001). Esta é a maior família de hepáticas nas florestas tropicais, com 285 espécies registradas no Brasil, e também a que detém o maior número de espécies endêmicas (Gradstein \& Costa 2003; Costa \& Peralta 2015).

As espécies de Lejeuneaceae identificadas estão distribuídas em 13 gêneros, sendo Cheilolejeunea e Lejeunea os mais representativos. Lejeunea flava foi a hepática mais frequente, com 75 ocorrências, ocorrendo também em canga aberta. É uma espécie tropical muito comum em árvores vivas e em rochas (Gradstein \& Costa 2003). Nas cangas da Serra dos Carajás, a espécie foi tipicamente coletada como corticícola e epíxila e, por ser generalista, era esperada a sua ocorrência em uma vegetação como a de canga.

Cheilolejeunea oncophylla destacou-se com 37 ocorrências em substratos como tronco vivo e rochas de ferro, corroborando com as observações de Grolle \& Reiner-Drehwald (1997), em ambientes como mata baixa sobre canga, canga

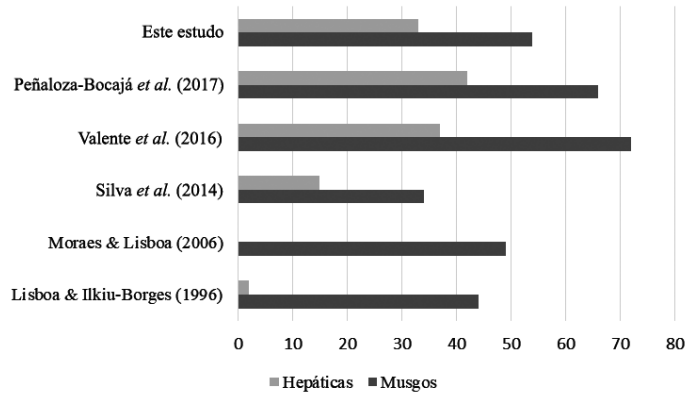

Figura 3 - Comparação entre a riqueza de espécies da canga da Serra dos Carajás e outros trabalhos realizados em áreas rupestres no Brasil.

Figure 3 - Comparison between the species richness of the canga from Serra dos Carajás and further studies from rupestrial areas in Brazil. aberta, próximo a campos brejosos, a margem de lagoas e córregos temporários de drenagem natural.

Dentre os musgos, os que mais se destacaram foram as espécies Campylopus savannarum e Microcalpe subsimplex, com 91 e 83 ocorrências, respectivamente. Campylopus savannarum é uma espécie muito comum em vegetação rupestre no Brasil e apresenta uma variação morfológica muito grande (Frahm 1991). Nos trabalhos de Lisboa \& Ilkiu-Borges (1996) e Moraes \& Lisboa (2006), assim como em outros trabalhos em áreas rupestres no Brasil, C. savannarum foi bastante frequente. $\mathrm{Na}$ área de estudo, essa espécie ocorreu em todas as serras e cangas amostradas, principalmente sobre rocha de ferro em canga aberta com muita exposição solar. Microcalpe subsimplex também é bem distribuída em todo o Brasil, ocorrendo em todos os tipos de ecossistemas, formando grandes tapetes sobre troncos vivos, troncos mortos e solo (Florschütz-de-Waard 1996; Costa \& Peralta 2015).

Das espécies identificadas, $51,4 \%$ ocorreram sobre árvore viva (corticícolas), seguido de 33\% sobre rocha de ferro (rupícolas), $8,8 \%$ sobre tronco em decomposição (epíxilas), 4,6\% sobre solo (terrícolas), 1,6\% sobre cupinzeiro e apenas $0,6 \%$ ocorreram sobre folha (epífilas).

A predominância de espécies corticícolas deu-se principalmente pela presença de árvores e arbustos em mata baixa sobre canga, que oferecem sombra e umidade para a colonização dessas comunidades mais vulneráveis a exposição solar e dessecação. Outro substrato importante (e abundante) é o rupestre, dada a própria natureza da canga.

Durante as expedições foi observada uma grande quantidade de espécimes de briófitas, principalmente Leucobryaceae, Calymperaceae e Bartramiaceae, ocorrendo diretamente sobre as rochas de ferro em canga aberta, expostas à radiação solar. Algumas espécies não esperadas em canga aberta, como membros de Fissidentaceae, foram comuns nesse ambiente, entretanto, colonizando locais úmidos e pouco expostos como base e frestas de rochas ou ainda mais protegidos, como espaços sob as rochas. De acordo com Gradstein et al. (2001), essa família pode ocorrer em locais abertos, desde que com umidade suficiente. As espécies Cololejeunea cardiocarpa, Drepanolejeunea fragilis e Lejeunea flava foram as únicas coletadas sobre folha.

Das Serras estudadas, a Serra Norte apresentou 27 espécies exclusivas, sendo a maioria composta por musgos, enquanto a Serra 
Sul apresentou 12 espécies exclusivas, a maioria composta por hepáticas. A canga da Mina N2 foi a mais rica dentre as cangas da Serra Norte e a $\mathrm{S} 11 \mathrm{C}$ foi a mais rica dentre as cangas da Serra Sul. Tanto a Mina N2 quanto a S11C apresentam grande quantidade de arbustos e árvores, substrato preferencial das briófitas de Carajás. A canga S11C, mais especificamente, abriga uma lagoa de macrófitas e um córrego temporário de drenagem natural, o que torna o ambiente mais favorável para a colonização de um maior número de espécies.

As briófitas foram praticamente ausentes na canga da Serra da Bocaina, pois a mesma havia sofrido uma queimada semanas antes das coletas. O único exemplar foi a espécie Campylopus savannarum que estava sobre rocha de ferro exposta e estava rebrotando a partir de restos de um tufo carbonizado.

Quanto à guildas de tolerância, predominaram as espécies generalistas (36 spp.), seguidas das epífitas de sol (23 spp.) e sombra (20 spp.). Dez espécies não foram classificadas em nível de grupo ecológico. As espécies neotropicais predominaram nas áreas de canga (46 spp.), seguidas das de distribuição pantropical (18 spp.), com ampla distribuição (10 spp.), distribuição disjunta (6 spp.), nas Américas (4 spp.) e América do sul (4 spp.).

As espécies Calymperes lonchophyllum, Fissidens pellucidus, Fissidens radicans, Orthorrhynchidium planifrons, Rhodobryum subverticillatum, Rosulabryum capillare, Trichosteleum lonchophyllum e Trichosteleum papillosum depositadas no Herbário MG e registradas nos trabalhado de Lisboa \& IlkiuBorges (1996) e Moraes \& Lisboa (2006) não foram encontradas nas expedições recentes realizadas nas cangas da Serra dos Carajás.

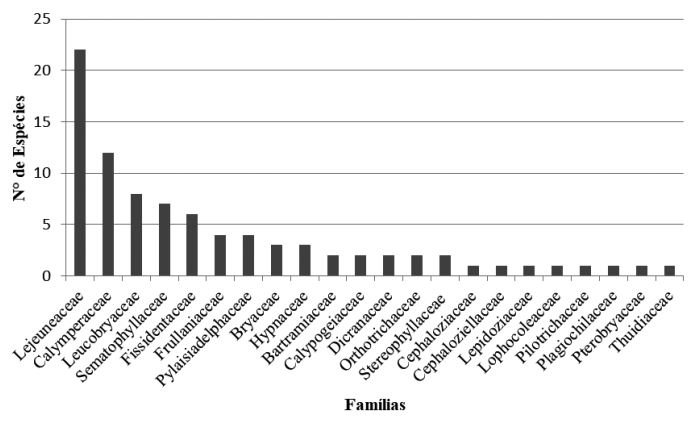

Figura 4-Riqueza de espécies por famílias identificadas para as áreas de canga da Serra dos Carajás, Pará, Brasil. Figure 4 - Species richness by families identified for the canga areas of Serra dos Carajás, Pará, Brazil.
Todavia, este projeto acrescentou 51 espécies de briófitas para o que já se conhecia das áreas canga de Carajás, entre elas 17 espécies de musgos e 34 espécies de hepáticas. Campylopus heterostachys, Frullania intumescens, Lejeunea angusta, Lophocolea bidentata, Schiffneriolejeunea amazonica, Telaranea nematodes e Vitalia cuspidifera foram novas ocorrências para o estado do Pará (Lima et al. 2018; Ilkiu-Borges \& Oliveirada-Silva 2016b, 2017c, 2018; Oliveira-da-Silva et al. 2018). Cephaloziopsis intertexta, Lejeunea oligoclada e Vitalia caespitosa foram registradas pela primeira vez na região Norte (Ilkiu-Borges \& Oliveira-da-Silva 2017b, 2018; Oliveira-da-Silva \& Ilkiu-Borges 2018) e Campylopus tallulensis foi novo registro para o Brasil (Oliveira-da-Silva et al. 2018).

As excussões deste projeto até as cangas da Serra dos Carajás ocorreram de 2015 a 2017 , e o início das publicações, com primeiro volume especial dedicado à Flora das cangas da Serra dos Carajás, aconteceu no ano de 2016. Dito isso, Calypogeia miquelii, Frullania riojaneirensis e Metalejeunea cucullata foram registradas posteriormente a publicação ou submissão da flora de suas famílias (Ilkiu-Borges \& Oliveira-da-Silva 2016a, Lima et al. 2018; Ilkiu-Borges \& Oliveirada-Silva 2018).

\section{Conclusão}

O estudo das briófitas na Serra dos Carajás possibilitou o conhecimento de 51 novos registros para as cangas, sete novas ocorrências para o estado do Pará, três novos registros para a região Norte e um novo registro para o Brasil. A canga na Serra dos Carajás apresentou uma grande riqueza de briófitas, assim como sua composição está de acordo com o esperado para áreas rupestres na Amazônia, salientando a elevada riqueza de especialistas de sombra. A presença dessas especialistas em área com grande exposição solar e sujeitas a dessecação, demonstraram que as poucas árvores da canga assim com as pequenas áreas de mata baixa que formam ilhas de vegetação ou ainda nichos específicos como frestas e base de rochas ou espaços sob rochas, servem como refúgio para essas espécies.

\section{Agradecimentos}

Agradecemos ao Museu Paraense Emílio Goeldi e Instituto Tecnológico Vale, a infraestrutura e demais apoios fundamentais para o 
desenvolvimento deste trabalho, assim como à Dra. Ana Maria Giulietti Harley e ao Dr. Pedro Viana, coordenadores do projeto conveniado MPEG/ITV/ FADESP (01205.000250/2014-10) e ao projeto aprovado pelo CNPq (processo 455505/2014-4), o financiamento; ao ICMBio, em especial ao biólogo Frederico Drumond Martins, a licença de coleta concedida e suporte nos trabalhos de campo; ao CNPq, a bolsa de Mestrado concedida ao primeiro autor e a bolsa de Produtividade em Pesquisa concedida à segunda autora.

\section{Referências}

BFG - The Brazil Flora Group (2015) Growing knowledge: an overview of seed plant diversity in Brasil. Rodriguésia 66: 1085-1113.

Buck WR (2003) Guide to the plants of Central French Guiana - part 3. Mosses. Memoirs of The New York Botanical Garden 76: 26-123.

Buck WR (2006) Plants of Central French Guiana. Mosses, New York 76: 43-222.

Cavalcante PB (1970) Centrosema carajasense, uma nova Leguminosae da Amazônia brasileira. Boletim do Museu Paraense Emílio Goeldi, série Botânica 37: 1-4

Costa DP \& Peralta DF (2015) Bryophytes diversity in Brazil. Rodriguésia 66: 10631071.

Costa DP, Pôrto KC, Luizi-Ponzo AP, Ilkiu-Borges AL, Bastos CJP, Câmara PEAS, Peralta DF, Bôas-Bastos SBV, Imbassahy CAA, Henriques DK, Gomes HCS, Rocha LM, Santos ND, Siviero TS, Vaz-Imbassahy TF \& Churchill SP (2011) Synopsis of the Brazilian moss flora: checklist, distribution and conservation. Nova Hedwigia 93: 3-4, 277-334.

Crandall-Stotler B; Stotler R \& Long D (2008) Morphology and classification of the Marchantiophyta.In: Goffinet B \& Shaw AJ. Bryophyte Biology. Cambridge University Press, Cambridge. Pp.1-54.

Crum HA \& Anderson LE (1981) Mosses of Eastern North America. Vol. 1. New York Columbia University Press, New York. Pp. 538-574.

Fagundes DN, Tavares-Martins ACC, Ilkiu-Borges AL, Moraes ENR \& Santos RCP (2016) Riqueza e aspectos ecológicos das comunidades de briófitas (Bryophyta e Marchantiophyta) de um fragmento de Floresta de Terra Firme no Parque Ecológico de Gunma, Pará, Brasil. Iheringia, série botânica 71: $72-84$.

Florschütz-de Waard J (1996) Sematophyllaceae. Musci III. In: Goerts-Van Rijn ARA.(ed.) Flora of the Guianas. Royal Botanical Gardens, Kew. Pp. 384-438.

Frahm JP (1991) Dicranaceae: Campylopodioideae, Paraleucobryoideae. Flora Neotropica 54: 1-238.

Goffinet B, Buck WR \& Shaw JA (2008) Morphology, anatomy, and classification of the Bryophyta.
In: Goffinet B \& Shaw AJ. Bryophyte Biology. Cambridge University Press, Cambridge. Pp. 55-138.

Golder Associates Brasil (2010) Projeto Ferro Carajás S11D / Estudos de Impacto Ambiental EIA/RIMA. No Golder: RT-079-515-5020-0029-02-J. Golder Associates Brasil Consultoria e Projetos Ltda, Belo Horizonte. Pp. 1-579

Gradstein SR \& Costa DP (2003) The Hepaticae and Anthocerotae of Brazil. Memoirs of the New York Botanical Garden 87:1-318.

Gradstein SR \& Ilkiu-Borges AL (2009) Guide to the plants of Central French Guiana. Part 4. Liverworts and Hornworts. Memoirs of The New York Botanical Garden 76: 1-140.

Gradstein SR, Churchill SP \& Salazar-Allen N (2001) Guide to the bryophytes of tropical America. Memoirs of the New York Botanical Garden 86: $1-577$.

Grolle R \& Reiner-Drehwald ME (1997) Cheilolejeunea oncophylla (Angstr.) Grolle \& Reiner comb. nov. (Lejeuneaceae), from Neotropics. Journal of Bryology 19: 781-785.

Ilkiu-Borges AL \& Oliveira-da-Silva FR (2016a) Flora das cangas da Serra dos Carajás, Pará, Brasil: Calypogeiaceae. Rodriguésia 67: 1129-1131.

Ilkiu-Borges AL \& Oliveira-da-Silva FR (2016b) Flora das cangas da Serra dos Carajás, Pará, Brasil: Lepidoziaceae. Rodriguésia 67: 1133-1135.

Ilkiu-Borges AL \& Oliveira-da-Silva FR (2017a) Flora das cangas da Serra dos Carajás, Pará, Brasil: Cephaloziaceae. Rodriguesia 68: 803-315.

Ilkiu-Borges AL \& Oliveira-da-Silva FR (2017b) Flora das cangas da Serra dos Carajás, Pará, Brasil: Cephaloziellaceae. Rodriguesia 68: 807-808.

Ilkiu-Borges AL \& Oliveira-da-Silva FR (2017c) Flora das cangas da Serra dos Carajás, Pará, Brasil: Lophocoleaceae. Rodriguesia 68: 817-818.

Ilkiu-Borges AL \& Oliveira-da-Silva FR (2017d) Flora das cangas da Serra dos Carajás, Pará, Brasil: Plagiochilaceae. Rodriguesia 68: 823-825.

Ilkiu-Borges AL \& Oliveira-da-Silva FR (2018) Flora das cangas da Serra dos Carajás, Pará, Brasil: Lejeuneaceae. Rodriguésia 69: 989-1012.

Lima E, Oliveira-da-Silva FR \& Ilkiu-Borges AL (2018) Flora das cangas da Serra dos Carajás, Pará, Brasil: Frullaniaceae. Rodriguésia 69: 973-981.

Lisboa RCL \& Ilkiu-Borges F (1996) Briófitas da Serra dos Carajás e sua possível utilização como indicadoras de metais. Boletim Museu Paraense Emílio Goeldi, série botânica 12: 161-181.

Lisboa RCL (1993) Musgos Acrocárpicos do estado de Rondônia. Coleção Adolpho Ducke. Boletim Museu Paraense Emílio Goeldi, Belém. Pp. 1-272.

Lisboa RCL (1994) Adições à brioflora do estado do Pará. Boletim Museu Paraense Emílio Goeldi série botânica 10: 15-42. 
Moraes ENR \& Lisboa RCL (2006) Musgos (Bryophyta) da Serra dos Carajás, estado do Pará, Brasil. Boletim do Museu Paraense Emilio Goeldi, série Ciências Naturais 1: 61-63.

Mota NFO, Silva LVC, Martins FD \& Viana PL (2015) Vegetação sobre sistemas ferruginosos da Serra dos Carajás. In: Carmo FF \& Kamino LHY (org.) Geossistemas Ferruginosos do Brasil: áreas prioritárias para conservação da diversidade geológica e biológica, patrimônio cultural e serviços ambientais. Belo Horizonte 289-315.

Oliveira-da-Silva FR \& Ilkiu-Borges AL (2016a) Flora das cangas da Serra dos Carajás, Pará, Brasil: Bartramiaceae. Rodriguésia 67: 1125-1128.

Oliveira-da-Silva FR \& Ilkiu-Borges AL (2016b) Flora das cangas da Serra dos Carajás, Pará, Brasil: Stereophyllaceae. Rodriguésia 67: 1137-1140.

Oliveira-da-Silva FR \& Ilkiu-Borges AL (2017a) Flora das cangas da Serra dos Carajás, Pará, Brasil: Bryaceae. Rodriguesia 68: 797-801.

Oliveira-da-Silva FR \& Ilkiu-Borges AL (2017b) Flora das cangas da Serra dos Carajás, Pará, Brasil: Fissidentaceae. Rodriguesia 68: 809-815.

Oliveira-da-Silva FR \& Ilkiu-Borges AL (2017c) Flora das cangas da Serra dos Carajás, Pará, Brasil: Pterobryaceae. Rodriguesia 68: 827-828.

Oliveira-da-Silva FR \& Ilkiu-Borges AL (2017d) Flora das cangas da Serra dos Carajás, Pará, Brasil: Orthotrichaceae. Rodriguesia 68: 819-822.

Oliveira-da-Silva FR \& Ilkiu-Borges AL (2018) Flora das cangas da Serra dos Carajás, Pará, Brasil: Sematophyllaceae. Rodriguésia 69: 1035-1044.

Oliveira-da-Silva FR, Ramalho AJ \& Ilkiu-Borges AL (2018) Flora das cangas da Serra dos Carajás, Pará, Brasil: Leucobryaceae. Rodriguesia 69: 1013-1024.

Peñaloza-Bocajá GF, Oliveira BA, Araújo CAT, Fantecelle LB \& Maciel-Silva AS (2017) Bryophyte reproduction on ironstone outcrops: delicate plants in harsh environments. Flora 238: 155-161.

Pursell RA (2007) Fissidentaceae. Flora Neotropica Monograph 101: 1-278.

Reese WD (1993) Calymperaceae. Flora Neotropica Monograph 58: 1-102.

Richards PW (1984) The ecology of the tropical forest bryophytes. In: Schuster RM (ed.) New Manual of Bryology. Vol. 2. The Hattori Botanical Laboratory, Nichinan. Pp. 1233-1270.

Santos ND, Costa DP, Kinoshita LS \& Shepherd GJ (2011) Aspectos brioflorísticos e fitogeográficos de duas formações costeiras de Floresta Atlântica da
Serra do Mar, Ubatuba/SP, Brasil. Biota Neotropica 11: 425-438.

Secco RS \& Mesquita AL (1983) Nota Sobre a Vegetação de Canga da Serra Norte. I. Boletim Paraense Emílio Goeldi, Nova série botânica 59: 1-13.

Sharp AJ, Crum H \& Eckel PM (1994) The moss flora of Mexico. Memoirs of The New York Botanical Garden 69: 1-1113.

Silva MFF (1991) Análise florística da vegetação que cresce sobre canga hematítica em Carajás-PA (Brasil). Boletim Paraense Emílio Goeldi, série especial 7: 79-108.

Silva MFF, Secco RS \& Lobo MGA (1996) Aspectos ecológicos da vegetação rupestre da Serra dos Carajás, estado do Pará, Brasil. Acta Amazonica 26: 17-44.

Silva TO, Silva MPP \& Pôrto KC (2014) Briófitas de Afloramentos Rochosos do estado de Pernambuco, Brasil. Boletim do Museu de Biologia Mello Leitão 36: 85-100.

Tavares ACC (2009) Florística e ecologia das comunidades de briófitas em florestas de terra firme no estado do Pará, Amazônia. Tese de Doutorado. Instituto de Pesquisas Jardim Botânico do Rio de Janeiro/Escola Nacional de Botânica Tropical, Rio de Janeiro. Pp. 1-132.

Valente EB, Pôrto KC \& Bastos CJP (2017) Habitat heterogeneity and diversity of bryophytes in campos rupestres. Acta Botanica Brasilica 31: 241-249.

Viana PL, Mota NFO, Gil ASB, Salino A, Zappi DC, Harley RM, Ilkiu-Borges AL, Secco RS, Almeida TE, Watanabe MTC, Santos JUM, Trovó M, Maurity C \& Giulietti AM (2016) Flora das cangas da Serra dos Carajás, Pará, Brasil: história, área de estudos e metodologia. Rodriguésia 67: 1107-1124

Visnadi RS (2013) Briófitas de áreas antrópicas do Parque Estadual da Serra do Mar, Núcleo Picinguaba, Ubatuba, estado de São Paulo, Brasil. Boletim do Museu Paraense Emílio Goeldi. Ciências Naturais, Belém 8: 49-62,

Yano O (1984) Briofitas. In: Fidalgo O \& Bononi VLR (eds.) Técnicas de coleta, preservação e herborização de material botânico. Séries Documentos. Instituto de Botânica, São Paulo. Pp. 27-30.

Yano O (2011) Catálogo de musgos brasileiros: literatura original, basiônimo, localidade-tipo e distribuição geográfica. Instituto de Botânica, São Paulo. 180p.

Yano O (1992) Leucobryaceae (Bryopsida) do Brasil. Tese de Doutorado. Universidade de São Paulo. São Paulo. 318p. 